\title{
How effective are treatments for anxiety after stroke? - A Cochrane Review summary with commentary
}

\author{
Peter Knapp \\ Department of Health Sciences and the Hull York Medical School, University of York, York, YO10 5DD, UK \\ E-mail: peter.knapp@york.ac.uk.
}

\begin{abstract}
The aim of this commentary is to discuss in a rehabilitation perspective the published Cochrane Review "Interventions for treating anxiety after stroke" by Knapp, Campbell Burton, Holmes, Murray, Gillespie, Lightbody, Watkins, Chun, $\&$ Lewis ${ }^{1}$, under the direct supervision of the Cochrane Stroke Group. This Cochrane Corner is produced in agreement with NeuroRehabilitation by Cochrane Rehabilitation.
\end{abstract}

\section{Background}

Stroke is the leading cause of adult disability. Anxiety is the most common mental health disorder. Anxiety after stroke has a prevalence of 20-25\% (Campbell Burton et al., 2013), and it can remain a common problem several years after stroke (Ayerbe et al., 2013). Distinct types of anxiety disorders are known, including general anxiety disorder (GAD), panic disorder, obsessive-compulsive disorder (OCD), social phobia, and post-traumatic stress disorder (PTSD), which share the features of excessive and irrational fear, subjective apprehension, and difficulty and distress in managing daily tasks, as well as physical, cognitive or behavioural symptoms.

\footnotetext{
${ }^{1}$ The abstract/plain language summary of this Cochrane Review is taken from a Cochrane Review previously published in the Cochrane Database of Systematic Reviews 2017, Issue 5. DOI: 10.1002/14651858.CD008860.pub3. (see www.cochrane library.com for information). Cochrane Reviews are regularly updated as new evidence emerges and in response to feedback, and the Cochrane Database of Systematic Reviews should be consulted for the most recent version of the review.
}

Co-morbidity of anxiety with depression after stroke is very high.

\section{Interventions for treating anxiety after stroke}

(Knapp, Campbell Burton, Holmes, Murray, Gillespie, Lightbody, Watkins, Chun, \& Lewis, 2017)

\section{What is the aim of this Cochrane Review?}

The aim of this Cochrane Review was to assess the effectiveness of pharmaceutical, psychological, complementary, or alternative interventions in treating stroke patients with anxiety disorders or symptoms.

\section{What was studied in the Cochrane Review?}

The population addressed in this review was stroke patients with anxiety symptoms or a clinical diagnosis of an anxiety disorder. The interventions studied were pharmaceutical and psychological treatments for stroke. The comparison interventions in 
the included trials varied (care as usual; combined pharmaceutical and psychological treatment). The outcomes studied were anxiety caseness, anxiety symptoms and quality of life.

\section{Up-to-dateness of the Cochrane Review}

The review authors searched for studies that had been published up to January 2017.

\section{What are the main results of the Cochrane Review?}

The review included three studies. The quality of the evidence in these studies was judged to be very low.

The review shows that:

- one small trial of a psychological intervention (based on relaxation) resulted in a lower anxiety symptom score in treated patients, compared to those who received care as usual.

- one small trial showed that patients who received an antidepressant alone, or an antidepressant and psychotherapy delivered in groups, had lower anxiety symptom scores than control group patients who received care as usual. Activities of daily living (indicated by Barthel score) increased in all three groups over the period of the trial, with greater increases in both the antidepressant and the antidepressant and psychotherapy groups.

- one small trial of an antidepressant showed that the anxiety symptom scores reduced in both the antidepressant group and the control group (who received care as usual), but the reduction was greater in the antidepressant group.

\section{How did the authors conclude on the evidence?}

The authors concluded that the available evidence was of low quality, with small numbers of participants and subject to high risk of biases. Statistical meta-analysis was not possible due to heterogeneity among the included studies. There is an urgent need for adequately sized, high quality trials to provide evidence for potential interventions for this important and impactful health problem.

\section{What are the implications of the Cochrane evidence for practice in neurorehabilitation?}

Both psychological and pharmaceutical interventions show promise for treating anxiety after stroke, but further trial evidence is needed before clinical practice recommendations can be made. There is some evidence that anxiety after stroke is associated with difficulties in managing activities of daily living (Campbell Burton et al., 2013) and so the treatment of anxiety and/or depression in stroke patients is important for rehabilitation professionals. Further research has the potential to change the evidence for clinical practice because of the low levels of certainty of the current evidence. Moreover, future research into anxiey after stroke should assess quality of life and caregiver burden outcomes since studies in this review did not evaluate these outcomes. From a rehabilitation perspective, detailed information on functional outcomes would be noteworthy. Only one study included in the review measured functional outcomes.

\section{Acknowledgments}

We thank Cochrane Rehabilitation and the Cochrane Stroke Group for reviewing the contents of the Cochrane Corner.

\section{Conflict of interest}

The author declares no conflicts of interest.

\section{References}

Ayerbe, L., Ayis, S. A., Crichton, S., Wolfe, C. D. A., \& Rudd, A. G. (2013). Natural history, predictors and associated outcomes of anxiety up to 10 years after stroke: The South London Stroke Register. Age and Ageing, 43(4), 542-547. doi: 10.1093/ageing/aft208.

Campbell Burton, C. A., Murray, J., Holmes, J., Astin, F., Greenwood, D., \& Knapp, P. (2013). Frequency of anxiety after stroke: A systematic review and meta-analysis of observational studies. International Journal of Stroke, 8(7), 545-559. doi: 10.1111/j.1747-4949.2012.00906.x

Knapp, P., Campbell Burton, C. A., Holmes, J., Murray, J., Gillespie, D., Lightbody, C. E., Watkins, C. L., Chun, H. Y., \& Lewis, S. R. (2017). Interventions for treating anxiety after stroke. Cochrane Database Systematic Reviews, 5, CD008860. doi: 10.1002/14651858.CD008860.pub3. 